\title{
Effects of dietary phytoestrogens on the developmental reprogramming of steroid-hormone influenced traits: Medicinal and evolutionary aspects
}

\author{
György Csaba* \\ Department of Genetics, Cell- and Immunobiology, Semmelweis University, Budapest, Hungary
}

\begin{abstract}
Nutritional phytoestrogens are weak estrogens, which entering into the human organism are able to reprogram the developmental program in sensitive periods of life (perinatally, at weaning, at puberty and in certain systems (e.g. immune system) during the whole life. Consequently they could cause (by faulty hormonal imprinting) lifelong alterations in the binding capacity of hormone receptors, inclination to diseases, manifestation of diseases etc. At the same time by adult age employment they can help healing of certain diseases. In some human cultures the consumption of phytoestrogens (isoflavones, as soy genistein or daidzein) is regular (in Asian countries) for thousand years, and this could contribute to the differences between the physiology of races. At present the consumption of soy (and isoflavones=phytoestrogens in them) is exponentially growing in European and American countries, what could influence the future (evolutionary) development (and uniformization) of endocrine (and endocrine-directed) systems of man. Some transformations of life-periods are observed (e.g. early menarche) and more are expected, however without serious attention. Considering this, phytoestrogens are the Troian horse of endocrine disruptors. Studying from medicinal aspect, the irregular (undosed) eating of phytoestrogens especially which does not attend the sensitive life-periods, can influence the endocrine system as well, as the strongly endocrine-regulated systems, which can be manifested in lower risk of later manifested diseases, as tumors or geriatric alterations however, it can cause reproductive and immune-deficiencies. The positive and mainly negative character of phytoestrogens have to be propagated for doctors and laymen alike. As phytoestrogens could cause different durable pathological alterations, the label on their presence (and effects?) in commercial foods, would be advisable.
\end{abstract}

The function of the mammalian (human) organism is regulated by the neuroendocrine system. The regulating humoral factors are the hormones, which are bound by hormone receptors. These structures which are localized either in the plasma membrane or inside the cell, recognize the hormones and transmit the hormone-beared information to the response-able organelles of the cell, which responds to the hormonal signal. However, in the human environment there are natural and man-made hormone-like molecules, which are also recognized and bound by these receptors, occupying the site for the physiological hormone (inhibiting its action) or mimicking the physiological hormone (inducing the response of the given cell). These molecules are named endocrine disruptors (EDs).

EDs had been present always in the human environment in natural form, as products of volcanic eruptions or forest-fires, and as edibleplant components (phytosteroids) however the amount and variants of man made arteficial forms have been growing in the last decades. This directed the attention to their (harmful?) effects and forefronted the study of natural endocrine disruptors, which are present in human foods. Legumes contain them in significant quantities, as soybeans, green beans and mung beans [1,2], as well, as flaxseed [3]. In Asian countries (as China, Japan, Korea) these legumes are integral parts of regular diet for centuries however they are consumed in a gradually growing but already excessive amount in the European-American countries, as processed soy products (miso, natto, soy milk, tofu) and -as it is very important- in baby formulas, instead of mother-milk or cow-milk [4]. In addition, soy is present in bred, cold cuts, ice creams and confectionery, practically in the everyday alimentation. As soy contains isoflavones (genistein, daidzein), the consumption of soy and its products is going hand in hand with the consumption of isoflavones, which are similar to 17-estradiol in structure and effects [5]. With the proliferation of vegan alimentation soy consumption and in the frame of this, isoflavone intake is growing.

Each man has genes which determine the species: man; however, each man is specific, containing genes or gene complexes characteristic only to it. Nevertheless, characteristic to a certain man different genes of the gene-pool are manifested in different organs (cells), for example intestinal cells (in general) have the function of digestion and brain cells have the function of cognition, memory etc. Each cell of a given organism contain the genes which are characteristic to the species and the individuum however, not genetic, but epigenetic factors determine what parts of the gene-pool are manifested in the given organ or cell, and when. This epigenetic regulation is responsible for the expression of genes and an epigenetic program is present, which determines what appears in the given situation. In this mechanism the methylation of cytosin nucleotids (methylation pattern) of DNA play the main role, executed by methylase enzymes. If the promoter of a gene is strongly

${ }^{*}$ Correspondence to: György Csaba, Department of Genetics, Cell- and Immunobiology, Semmelweis University, Budapest, Hungary, E-mail: csaba. gyorgy@med.semmelweis-univ.hu

Key words: isoflavones, soy, endocrine system, hormonal imprinting, faulty imprinting, perinatal period, puberty

Received: December 04, 2019; Accepted: December 27, 2019; Published: January 03,2020 
methylated it can not be manifested, demethylation makes possible the expression of genetic information. The program can be changed, consequently the functional activity, the collaboration between genes and functions can be also changed and in the regulation of this process hormones play the main roles [6]. This means that steroid-hormonelike isoflavones can influence the expression of the program [7].

Although the effect of soy (or other plant) isoflavones (e.g. genistein, daidzein) have important effect on cellular functions in any section of life, there are specific, hypersensitive sections, as the perinatal and weaning periods. In these periods the developmental window for reprogramming is open and in normal cases hormonal imprinting happens. Hormonal imprinting is a part of the program-setting, in case of the first encounter between the developing receptor and its target hormone, by which the normal receptor-hormone connection develops and is maintained for life [6]. If -instead of the physiological hormonehormone-like molecules (EDs) meet the receptor, faulty imprinting develops with life-long consequences [8,9], as inclination to diseases, manifestation of diseases etc. later, far (in time) from the provocation. This is the faulty hormonal imprinting which is a functional teratogen, without morphological (structural) alterations [7-9]. Isoflavones are strong (faulty) imprinters, what means that early-life soy exposure causes durable alterations in the endocrine (and endocrine-influenced) systems [10-12]. The perinatal consumption of isoflavones (in baby formula) imprints the developing hormone receptors with lifelong consequences.

Adolescence (puberty) is a long period of life, when the whole neuroendocrine system is endogeneously reprogrammed. This appears in the behavior of teen-agers as well as the structural transformation of the body and such systemes as the sexual sphere [13] etc. In this period the sensitivity to hormone-like molecules (imprinters), which can reprogram the epigenetic system, explosively increases which lifelong determines the further state of the organism (sexuality, behavior, habits). However, finishing adolescence also there is a possibility for provoking faulty hormonal imprinting in organs (systems) in which continuous differentation of cells is taking place, as the immune system $[14,15]$. Nevertheless, the main phase of reprogramming is the perinatal one, however, pubertal or later reprogramming could have similar effect with shorter validity.

\section{Positive and negative effects of phytoestrogens on the human organism}

Female cycling, sperm quality, menarche, prostate diseases are influenced by genistein, in animal and human cases alike $[16,17]$. Genistein activates tumor suppressor genes and affects cancer cell survival [18].

In animal (mice) experiments early-life exposure to genistein impairs fertility [19]. This due to the disruption of glucocorticoid signaling in imprinted animals. Genistein imprinting negatively impacts ovarian differentiation, estrus cyclicity [20], affects play behavior [21]. In animal experiments phytoestrogens reduced carcinogenesis, reproductive function, immune function and thyroid diseases [22].

Perinatal (early-life) genistein treatment in rat experiments enhanced later sexual activity of males and females alike [11]. Liver glucocorticoid receptor's density was reduced in adult male rats, after perinatal genistein imprinting [10]. Phytoestrogens can compensate sexual hormone deficiency in the menopause [23,24]. Aging brain (cognition) is negatively influenced over 65 years (12). In man occurrence of hypospadias was decreased by maternal intake of phytoestrogens [25]. Maternal (gestational) intake of soy showed high correletion with umbilical cord blood isoflavone content [26], however there were not associations between the dietary soy intake and maternal thyroid function [27].

Phytoestrogens have positive effects on depression and anxiety of menopausal women [28].

Genistein reduces (compensate) the harmful effects of bisphenol A exposure on rat prostate gland [29].

Resveratrol, the phytoestrogen of red vine improve cognitive function in postmenopausal vomen [30], can substitute conventional hormone therapy [31] and cause $25 \%$ reduction in tumor recurrence.

Soy isoflavone imprinting in mice increases the latency of spontaneous mammary tumors [32].

Perinatal phytoestrogen imprinting causes (in adults) higher bone mineral density (BMD) [33].

In man, lovered risks of menopausal symptoms, osteoporosis, cardiovascular diseases, obesity, metabolic syndrome, type 2 diabetes, brain function disorders (e.g. Alzheimer disease), breast cancer, prostate cancer, bowel cancer have been observed under the effect of phytoestrogens $[2,27,34]$.

\section{Discussion}

Studying the selected, however important data the impression is, that phytoestrogen interventions have both positive and negative influences [35], evaluated from medical point of view. However, from evolutiary aspect the judgement is different.

It seems to be likely that the neuroendocrine system of today's modern man is not the same as was many centuries or millenaries ago. A lot of chemical factors formed it, adapted to them as this was needed for the sustenance of human life. Nourishing factors was always important from this point of view, considering that the enteral route is the most suitable for intaking substances which are needed for the human organism, or can not be avoided though their danger was known. The human organism (similar to other less developed organisms) could adapt to the variable life conditions and this ensured the maintenace of its life. As an example: human organism is not able to produce vitamin C (which is produced by many non-human organism) however, this important molecule was obtained from outside, in the beginning unknowingly, later consciously. Considering the hormones: vitamin A (which is really an (exo)-hormone, is not produced by the human organism, but sufficient amount is intaked enterally and vitamin $\mathrm{D}$, which could be a hormone in the ancent periods of human life (in the sunburned Africa) and its production decreased by the northwandering of man; both became exohormones with receptors in the steroid receptor superfamily. This means that there is a well balanced collaboration between the human organism and its environment and what is absent inside the organism (and has vital importance) can be supplemented by exogeneous factors, which infiltrates into certain systems of the organism. It is possible that isoflavones are needed for some functions or for the avoidance of some impairments. In this case the uncertain effects of soy isoflavones to different processes indicates the infiltration of the molecules as exohormones and later the fixed function (endohormones=hormones) will develop [12]. It is very difficult to decide whether this process is adventageous or harmful as different aspects must be evaluated.

During evolution very long time is at the disposal of changing structurally or rather functionally and the modern man is the 
sequel of these changes. From this point of view, the distribution of phytoestrogens could be evaluated as positive inducers of the process. However, the effect of massive amount of phytoestrogens in a short time (by the transformation of nourishment) can be deleterious. In addition this transformation of nourishing-habits proceeds parallel with the appearance of industrial, communal, medical ED-contaminants supporting the harmful effects of each other. The nourishing factors construct an elevated basal ED-level on which the other EDs are deposited and together exceed the level which can be accepted by the human organism as a factor of healthy transformation, disturbing the normal slow (evolutionarily acceptable) transformation.

Man, as a species contains different races which represents different characters (for example European and Asian races are diffent in many indexes, as blood tension, time of menarche and menopause, aging), the transformation of nourishing can uniform these, disturbing the habitual incidence of diseases as well as the ability of defense. This could request the transformation of medical interventions.

Hormones are physiological molecules which transmits informations given by the central nervous system which are deciphered by different organs. However, natural and man-made synthetic hormones are used for substituting physiological ones or are used as medicaments in different diseases having well-controlled dosage. Phytoestrogens (isoflavones, as soy genistein or diadzein) have similar effects (26) however, as they are settled in foods, their dose can not be controlled, and this is especially problematic when infants are consuming them (in baby-formula) causing late manifested diseases, or other alterations $[36,37]$. At present there are not regulations determining the life-period and dose of phytoestrogen-containing nourishments, although this would be necessary, for avoiding overdosage and there is not descriptions which could designate the periods when special attention would be needed, for avoiding the late harmful effects. From this aspect the perinatal period is especially important (arteficial feeding included) however, also strongly sensitive the adolescent period and this must be considered. If we know that hormonal imprinting is not a time-dependent process but it is cell-differentiation dependent, the type of tissue (organ) must be also considered. For example, the cells of the immune system are differentating during the whole life, so phytoestrogens can imprint them at any time with lifelong consequences: autoimmunity, allergy, lifespan etc.

A special problem is the direct, maternally transmitted effect of phytoestrogens, as the maternal consumption of isoflavone-containing foods is hardly avoidable. In this case there are evidences that the phytoestrogens enter into the embryo or fetus [26,27], however late harmful effects (caused by faulty imprinting) were not observed, as this problem has not been studied thoroughly.

Advertisements suggest in tv and newspapers, or on the package of the goods, that some foods are lactose-free or gluten-free, consequently they are recommended for people who are sensitive to these materials, and this is reasonable, as glutene or lactose can provoke symptoms instantly in man, who are sensitive to them [38,39]. However, there are not ads, which announce a food as "phytoestrogen containing"” or "phytoestrogen-free”. The effect of phytoestrogens is not instant, but late manifested effects are provoked by them (by faulty hormonal imprinting) however, these effects could be more serious than that of gluten or lactose-provoked ones. Considering the above-mentioned facts the avoidance of isoflavones (combined with their targeted use) could be also important, possibly more important than those which are already advertised in this manner. Use of some sexual hormone preparations are forbidden in animal husbandry because of their possible intervention into the human organism, but use of soy as components of different foods is not considered. This call attention to the necessity of change in mentality of health protection in case of foodcontrol.

\section{Conclusions}

It seems to be very difficult to declare the general benefit or harm of phytoestrogens as both sides have evidences and both sides prevail in our everyday life as well, as in our present and future evolution. The opinions on benefits are uncertain and the future can not be forecasted. However- and this seems to be sure- impossible to negligate their effects in case of the manifestation of diseases, on the healing or aggravation of them and on the distribution to the effects of other disease-causing factors. In addition, human functional-biological evolution likely has not been finished. As the above-mentioned vitamins (exohormones) which was exogeneous factors earlier and merged into the human organism [37], without which the human physiology would not be complete, phytoestrogens also could be requested and merged, regulating such life-processes which are at present without concrete regulator. As an example: Systematic consumption of phytoestrogens at early life influences the time of menarche (the time of beginning of adolescence) and their infiltration into the endogeneous hormonal system could influence this (and parallel sexual functions) regularly. It is not known but imaginable that more sexual hormones (estrogens) will be requested in the future, for the normal reproduction of man and phytoestrogens will solve this problem. Nevertheless, this only a product of phantasy, without thorough theoretical or practical basis. However, it is not phantasmagory, that phytoestrogen-rich food can substitute synthetic medicaments, influencing (healing) certain diseases, and some hormone-like materials (isoflavones) decrease the risk of such diseases which are caused mainly by our modern life-form and this latter attracts the infiltration of them into the human organism after thousand years of tenure. Presently phytoestrogens have not receptors of their own and they are using the estrogen receptors for expressing their effects, however it is not impossible that they will have separate receptors in the future, which will be independent on endogeneous estrogens. In this latter case their effects could be well differentiated from the endogeneous relatives.

At present, man-made molecules are in the forefront of studies on the harmful and beneficial effects of endocrine disruptors, whereas phytoestrogens are consumed in an extreme and growing amount. The explanation could be the experience, that more than one milliard people consume them in Asia without rampageous complaints. However, during the passing time, phytoestrogens crookedly changes (reprogram) the onset of menarche (a very important developmental event) in the European and American populations [40-42], which is a part and signal of sexual maturation, in relation with other sexual factors and likely together with other non-rampageous indexes. Whilst the official and medical attentions are concentrated on the effects of industrial and agrotechnical materials, the effects of phytoestrogens are almost neglected, although they are the Troian horse of endocrine disruptors.

\section{References}

1. Zaheer K, Humayoun Akhtar M (2017) An updated review of dietary isoflavones: Nutrition, processing, bioavailability and impacts on human health. Crit Rev Food Sci Nutr 57: 1280-1293.

2. Patisaul HB, Jefferson W (2010) The pros and cons of phytoestrogens. Front Neuroendocrinol 31: 400-419. 
3. Dixon R (2004) Phytoestrogens. Ann Rev Plant Biol 55: 225-251.

4. Irvine CH, Fitzpatrick MG, Alexander SL (1998) Phytoestrogens in soy-based infant foods: concentrations, daily intake, and possible biological effects. Proc Soc Exp Biol Med 217: 247-253

5. Rietjens IMCM, Louisse J, Beekmann K (2017) The potential health effects of dietary phytoestrogens. Br J Pharmacol 174: 1263-1280.

6. Csaba G (2011) The biological basis and clinical significance of hormonal imprinting, an epigenetic process. Clin Epigenetics 2: 187-196.

7. Csaba G (2016) The faulty perinatal hormonal imprinting as functional teratogen. Curr Pediatr Rev 12: 222-229.

8. Csaba G (1984) The present state in the phylogeny and ontogeny of hormone receptors. Horm Metab Res 16: 329-335.

9. Csaba G (2008) Hormonal imprinting: phylogeny, ontogeny, diseases and possible role in presen-day human evolution. Cell Biochem Funct 26: 1-10.

10. Csaba G, Inczefi-Gonda Á (2002) Effect of a single treatment (imprinting) with genistein or combined treatment with genistein + benzpyrene on the binding capacity of glucocorticoid and estrogen receptors of adult rat. Hum Exp Toxicol 21: 231-234.

11. Csaba G, Karabélyos C (2002) Effect of single neonatal treatment with soy bean phytosteroid, genistein on the sexual behavior of adult rats. Acta Physiol Hung 89: 463-470.

12. Csaba G (2018) Effects of early endocrine disruptor exposures (faulty hormonal imprinting) on immunity. Austin Immunol 3: 1016-1022.

13. Csaba G (2017) The present and future of human sexuality: Impact of faulty hormonal imprinting. Sex Med Rev 5: 163-169.

14. Csaba G (2014) Immunoendocrinology: Faulty hormonal imprinting in the immune system. Acta Microbiol Immunol Hung 61: 89-106.

15. Csaba G (2017) The crisis of the hormonal system: the health-effects of endocrine disruptors. Orv Hetil 158: 1443-1451.

16. Ho SM, Cheong A, Adgent MA, Veevers J, Suen AA, et al. (2017) Environmental factors, epigenetics, and developmental origin of reproductive disorders. Reprod Toxicol 68: 85-104.

17. Cederroth CR, Zimmermann C, Nef S (2012) Soy, phytoestrogens and their impact on reproductive health. Mol Cell Endocrinol 355: 192-200.

18. Zhang Y, Chen H (2011) Genistein, an epigenetic modifier during cancer prevention. Epigenetics 6: 881-891.

19. Whirledge SD, Kisanga EP, Oakley RH, Cidlowski JA (2018) Neonatal genistein exposure and glucocorticoid signaling in the adult mouse uterus. Environ Health Perspect 126: 047002 .

20. Jefferson WN, Williams CJ (2011) Circulatig levels of genistein in the neonate, apart from dose and route, predict future adverse female reproductive outcomes. Reprod Toxicol 31: 272-279.

21. Flynn KM, Ferguson SA, Delclos KB, Newbold RR (2000) Effects of genistein exposure on sexually dimorphic behaviors in rats. Toxicol Sci 55: 311-319.

22. Tuohy PG (2003) Soy infant formula and phytoetrogens. J Paediatr Child Health 39: 401-405.
23. Jargin SV (2014) Soy and phytoetrogens: possible side effects. Ger Med Sci 12.

24. Diel P, Kurrat A, Oden C, Hanke L (2017) Risk and benefit of nutritional supplement for the treatment of postmenopausal complaints. Bundesgesundheitsblatt 60: 297-304.

25. Carmichael SL, Cogswell ME, Ma C, Gonzalez-Feliciano A, Olney RS, et al. (2013) Hypospadias and maternal intake of phytoestrogens. Am J Epidemiol 178: 434-440.

26. Nagata C, Iwasa S, Shimizu H (2006) Associations among maternal soy intake isoflavone levels in urine and blood samples, and maternal and umbilical hormone concentrations (Japan). Cancer Causes Control 17: 1107-1113.

27. Li J, Teng X, Shan Z (2011) Effects of dietary soy intake on maternal thyroid functions and serum anti-thyroperoxidase antibody level during early pregnancy. $J$ Med Food 14: $543-550$.

28. Fattah A (2017) Effect of phytoestrogen on depression and anxiety in menopausal women: A systematic review. J Menopausal Med 23: 160-165.

29. Bernardo BD, Brandt JZ, Grassi TE, Silveira RT, Scarano WR, et al. (2015) Genistein reduces the noxious effects of in utero bisphenol A exposure on the rat prostate gland at weaning and in adulthood. Food Chem Toxicol 84: 64-73.

30. Thaung Zaw JJ, Howe PRC, Wong RHX (2017) Does phytoestrogen supplementation improve cognition in humans? A systematic review. Ann NY Acad Sci 1403: 150-163.

31. Messina M (2014) Soy foods, isoflavones, and the health of postmenopausal women Am J Clin Nutr 100: 423S-430S.

32. Jin Z, MacDonald RS (2002) Soy isoflavones increase latency of spontaneous mammary tumors in mice. $J$ Nutr 132: 186-190.

33. Mardon J, Mathey J, Kati-Coulibaly S, Puel C, Davicco MJ, et al. (2008) Influence of lifelong soy isoflavones consumption on bone mass in the rat. Exp Biol Med 233: 229-237.

34. Lu Y, An Y, Xiao R (2018) Dietary soybean isoflavones in Alzheimer's disease prevention. Asia Pac J Clin Nutr 27: 946-954.

35. Peirotén A, Bravo D, Landete J (2019) Bacterial metabolism as responsible of beneficial effects of phytoestrogens on human health. Clin Rev Food Sci Nutr 4: 1-16.

36. Csaba G (2018) Effect of endocrine disruptor phytoestrogens on the immune system: present and future. Acta Microbiol Immunol Hung 65: 1-14.

37. Csaba G (2017) Vitamin-caused faulty perinatal hormonal imprinting and its consequences in adult age. Physiol Int 104: 217-225.

38. Czaja-Bulsa G (2015) Non-coeliac gluten sensitivity - a new disease with glutenintolerance. Clin Nutr 34: 189-194.

39. Szilagyi A (2015) Adult lactose digestion status and effects of disease. Can J Gastroenterol Hepatol 29: 149-156.

40. Marks KJ, Hartman TJ, Marcus M (2017) Exposure to phytoestrogens in utero and age at manarche in a contemporary British cohort. Environ Res 155: 287-293.

41. Parent AS, Franssen D, Bourguignon JP (2016) Current changes in pubertal timing: revised vision in relation with environmental factors including endocrine disruptors. Endocr Dev 29: 174-184

42. Mouritsen A, Aksglaede I, Juul A (2010) Hypothesis: exposure to endocrine-disrupting chemicals may interfere with timing of puberty. Int J Androl 33: 346-359.

Copyright: (C2020 Csaba G. This is an open-access article distributed under the terms of the Creative Commons Attribution License, which permits unrestricted use, distribution, and reproduction in any medium, provided the original author and source are credited. 